\title{
Red meat from animals offered a grass diet increases platelet $n-3$ PUFA in healthy consumers
}

\author{
A. J. Mcafee ${ }^{1}$, E. M. Mcsorley ${ }^{1}$, G. J. Cuskelly ${ }^{2}$, A. M. Fearon ${ }^{3}$, B. W. Moss ${ }^{3}$, J. A. M. Beattie ${ }^{3}$, \\ J. M. W. Wallace ${ }^{1}$, M. P. Bonham ${ }^{1}$ and J. J. Strain ${ }^{1}$ \\ ${ }^{1}$ Northern Ireland Centre for Food and Health, University of Ulster, Coleraine BT52 1SA, UK, ${ }^{2}$ Queens University, \\ Institute of Agri-Food and Land Use, School of Biological Sciences, Belfast BT9 5AG, UK and ${ }^{3}$ Agri-Food and \\ Biosciences Institute (AFBI), Belfast BT9 5PX, UK
}

Red meat produced from grass-fed animals, compared with that produced from concentrate-fed animals, contains increased concentrations of long chain (LC) $n-3$ polyunsaturated fatty acids (PUFA) ${ }^{(1,2)}$. The LC $n-3$ PUFA, eicosapentaenoic acid (EPA) and docosahexaenoic acid (DHA) are recommended in dietary quantities of $\geq 450 \mathrm{mg} / \mathrm{d}$ for the prevention of cardiovascular disease $(\mathrm{CVD})^{(3)}$. The aim of this study was to compare the effects of consuming red meat produced from either grass-fed animals, or concentrate-fed animals on platelet LC $n-3$ PUFA status.

A randomised, double-blind, dietary intervention study was carried out for 4 weeks on two groups of healthy male and female subjects, who replaced their habitual red meat intake with three portions per week of red meat produced from animals offered a finishing diet of either grass ( $n=18$ consumers) or concentrate $(n=20$ consumers). Meat was sourced from beef cattle $(n=8)$ and lambs $(n=44)$ offered a finishing diet of strictly grass or concentrate for a period of 6 weeks prior to slaughter. The weekly meat portions for consumers included one sirloin steak $(200 \mathrm{~g})$, one portion of mince beef $(250 \mathrm{~g})$ and four lamb medallions $(240 \mathrm{~g})$. Dietary intake, blood pressure, platelet fatty acid composition and serum lipids and lipoproteins were analysed at baseline and post-intervention.

Consumer platelet concentrations of EPA, docosapentaenoic acid (DPA), DHA and total LC $n-3$ PUFA were significantly higher in those subjects who consumed red meat from grass-fed animals compared to those who consumed red meat from concentrate-fed animals $(P<0.05)$. No significant differences in concentrations of serum cholesterol, triacylglycerols (TAG) or blood pressure were observed between groups.

The consumption of red meat, in amounts below the weekly limit recommended by the World Cancer Research Fund ${ }^{(4)}$, from grass-fed animals compared to concentrate-fed animals significantly increased consumer platelet LC $n-3$ PUFA status. Therefore, red meat from grass-fed animals may contribute appreciable amounts of dietary LC $n-3$ PUFA in populations where red meat is habitually consumed, which in turn would be beneficial for human health. Further research is needed about on-farm conditions to optimise grass-fed meat on a financially feasible commercial basis.

\begin{tabular}{|c|c|c|c|c|c|c|c|c|c|}
\hline \multirow[b]{2}{*}{ Fatty acids ( $\%$ total) } & \multicolumn{4}{|c|}{ Grass group $(n=18)$} & \multicolumn{4}{|c|}{ Concentrate group $(n=20)$} & \multirow[b]{2}{*}{$P^{*}$} \\
\hline & Baseline & SD & Post-intervention & SD & Baseline & SD & Post-intervention & SD & \\
\hline C20:5n-3 (EPA) & 4.96 & 1.15 & 5.75 & 0.81 & 4.88 & 0.86 & 4.58 & 1.31 & $* *$ \\
\hline $\mathrm{C} 22: 5 n-3$ (DPA) & 6.61 & 1.53 & 7.67 & 1.07 & 6.49 & 1.15 & 6.43 & 1.35 & ** \\
\hline $\mathrm{C} 22: 6 n-3$ (DHA) & 4.96 & 1.14 & 5.75 & 0.81 & 4.93 & 0.79 & 4.58 & 1.45 & ** \\
\hline LCn-3 PUFA & 16.52 & 5.52 & 19.19 & 3.11 & 16.31 & 3.27 & 15.59 & 3.11 & * \\
\hline$n-6: n-3$ & 0.83 & 0.36 & 0.63 & 0.09 & 0.82 & 0.35 & 0.8 & 0.12 & $* * *$ \\
\hline
\end{tabular}

*Difference between groups at post-intervention with baseline value as covariate in ANCOVA, where: $* P<0.05, * * P<0.01, * * * P<0.001$, NS, non significant $P>0.05$.

1. Aurousseau et al. (2004) Meat Sci 66(3), 531-541.

2. French et al. (2000) J Anim Sci 78, 2849-2855.

3. SACN/COT (Scientific Advisory Committee on Nutrition and Committee on Toxicity) (2004) Norwich: The Stationery Office.

4. WCRF (World Cancer Research Fund) (2007) Washington DC: American Institute for Cancer Research. 